\title{
Selective Primary Alcohol Oxidation of Lignin Streams from Butanol-Pretreated Agricultural Waste Biomass
}

\author{
Isabella Panovic, ${ }^{[a]}$ Christopher S. Lancefield, ${ }^{[a]}$ Darren Phillips, ${ }^{[b]}$ Mark J. Gronnow, ${ }^{[b]}$ and Nicholas J. \\ Westwood ${ }^{*[a]}$
}

\begin{abstract}
Chemically modified lignins are important for the generation of biomass-derived materials and as precursors to renewable aromatic monomers. A butanol-based organosolv pretreatment has been used to convert an abundant agricultural waste product, rice husks, into a cellulose pulp and three additional product streams. One of these streams, a butanol-modified lignin, was oxidised at the $y$ position to give a carboxylic acid functionalized material. Subsequent coupling of the acid with aniline aided lignin characterization and served as an example of the flexibility of this approach for grafting side chains onto a lignin core structure. The pretreatment was scaled up for use on a multi-kilogram scale, a development that enabled the isolation of an anomeric mixture of butoxylated xylose in high purity. The robust and scalable butanosolv pretreatment has been developed further and demonstrates considerable potential for the processing of rice husks.
\end{abstract}

\section{Introduction}

Lignocellulosic biomass consists of a mixture of cellulose (c.a. 30$50 \%$ ), lignin (c.a. $10-30 \%$ ) and hemicellulose (c.a. 20-35\%). ${ }^{[1]}$ Currently, the Kraft and lignosulfonate processes deliver large quantities $^{[2,3]}$ of valuable saccharide product streams at the required high purity levels. These methods also give a lignin stream which is typically burnt on-site to return energy back into the process or is used in low value materials as an additive or binding agent. ${ }^{[4]}$ There is considerable interest in higher value uses for lignin, due to its aromatic-rich structure, with research dominated by two main approaches; (i) its use in polymeric materials for co-polymer blending and grafting applications, ${ }^{[5,6]}$ and (ii) its depolymerisation to useful aromatic monomers. ${ }^{[7-9]}$ The structural complexity of lignin offers both a challenge and an opportunity. In theory a wide range of different feedstock

[a] Miss I. Panovic, Dr. C. S Lancefield, Dr N. J. Westwood School of Chemistry \& Biomedical Sciences Research Complex, University of St Andrews and EaStCHEM,

North Haugh, St Andrews, Fife (UK) KY16 9ST

E-mail: njw3@st-andrews.ac.uk

[b] Mr D. Phillips, Dr M. J. Gronnow

Biorenewables Development Centre,

1 Hassacarr Close, Chessingham Park, Dunnington, York (UK) YO19 5SN

Supporting information for this article is given via a link at the end of the document. chemicals and functional materials could be generated from lignin if suitable processes can be found.
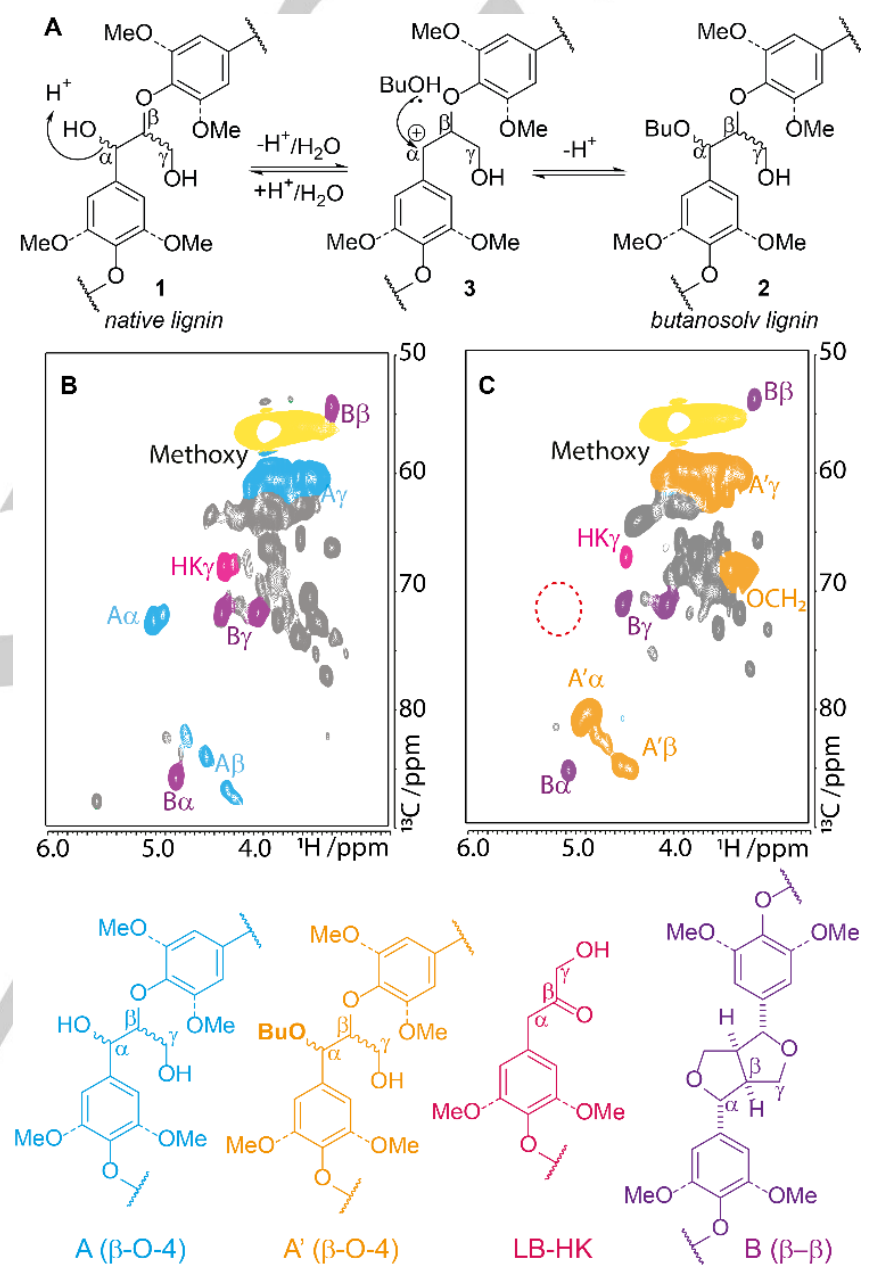

Figure 1. A Comparison of dioxasolv and butanosolv lignins. A Mechanism of formation of butoxylated $\beta-\mathrm{O}-4$ units during the butanosolv process. In an acidic organosolv pretreatment, benzylic cation 3 is formed from native $\beta-0-4$ unit 1 . For butanosolv, 3 is convert to a butoxylated $\beta-O-4$ unit 2 . In contrast for dioxasolv, 3 reacts to form condensed $\mathrm{C}-\mathrm{C}$ bond containing units and ligninbound Hibbert's ketones (LB-HK). ${ }^{[10]} \mathbf{B}$ and C 2D HSQC NMR $(700 \mathrm{MHz}$, d6DMSO) analysis of $\mathbf{B}$ dioxasolv walnut shell lignin and $\mathbf{C}$ butanosolv walnut shell lignin. Full conversion to the butoxylated $\beta-O-4$ unit 3 (orange signals) often occurs in the butanosolv process, as shown by the complete loss of the crosspeaks (highlighted in dashed red circle) corresponding to the native $\beta-O-4$ unit 1 (blue signals).

Most biorefineries focus on the carbohydrate product streams, meaning severe conditions are used to remove the "contaminating" lignin. Typically the lignin's $\beta-\mathrm{O}-4$ unit (structure 
1 in Figure $1 \mathrm{~A}$ ), which is the most abundant unit in native lignins, is heavily degraded. Destruction of the $\beta-O-4$ unit and extensive formation of additional $\mathrm{C}-\mathrm{C}$ bonds (condensation) yields lignins that are less chemically tractable than the native biopolymer. ${ }^{[11,12]}$ In contrast, some organosolv pretreatments treat lignocellulosic biomass $^{[13,14]}$ more gently with the drawback that less pure carbohydrate streams are formed. In addition, due to the frequent use of acidic conditions, some organosolv lignins are still condensed. We ${ }^{[15]}$ and others ${ }^{[16-20]}$ have previously reported the use of biorenewable butanol as a pretreatment solvent for woodybiomass sources, using both hardwoods and softwoods. ${ }^{[15]}$ Under the acidic butanosolv conditions, the hydroxyl at the $\alpha$-position of the $\beta-O-4$ unit is replaced by butanol leading to stabilisation of the $\beta-O-4$ unit in a "protected"[21] form (structure 2, Figures 1 and S1). Failure to trap benzylic cations such as 3 results in condensation or the formation of (lignin-bound) Hibbert's ketones (LB-HK, Figure 1). ${ }^{[10]}$ Another example of a "protected lignin" was reported recently by Luterbacher et al. who created a aldehyde-stabilised lignin in which the $\beta-O-4 \alpha$ - and $y$-hydroxyls were tethered together (double protection). ${ }^{[22,23]}$

In agriculture the majority of crops produced come from herbaceous monocots, such as rice, wheat, sugarcane and maize and therefore large volumes of agricultural waste from these sources are generated annually. Among these, rice is one of the most cultivated crops in the world with around 680 million tonnes produced per annum. ${ }^{[24]}$ Rice husks make up around $20 \mathrm{wt} \%$ of the rice grain and are composed of approximately $22 \%$ lignin, $38 \%$ cellulose, $18 \%$ hemicelluloses, $2 \%$ extractives and $20 \%$ ashes with variations depending on geographical origin. ${ }^{[25,26]}$ As a result of high lignin and ash content, the husks are not a viable animal feed, ${ }^{[27]}$ but they can be burnt to yield large quantities of silica that can be used in a number of material applications. ${ }^{[25,27-}$ 33] However, compared to the relatively well valorised polysaccharide fractions and processing of rice husk-derived silica, little attention has been paid to the abundant lignin fraction.

Here, we report that a butanol-based organosolv pretreatment of rice husks allows for efficient processing of the biomass. We show that this process is scalable up to a multikilogram scale and that the resulting rice husk butanosolv lignin (RHBL) has high $\beta-\mathrm{O}-4$ content with the expected incorporation of butanol at the $\alpha$-position of the $\beta-O-4$ unit. RHBL is shown to be a suitable substrate in an organocatalytic oxidation to give a $\gamma$ carboxylic acid-containing lignin that can be used in amide coupling reactions. Butoxylated xylose (mixture of anomers) is also isolated in high purity from a hemicellulose-derived stream.

\section{Results and Discussion}

Previously we have described the application of butanosolv pretreatment of beech (hardwood), walnut shells (hardwood endocarp) and Douglas fir (softwood) (Table S1, entries 1-3, Figure S4). ${ }^{[15]}$ In each case the cellulose was retained in a pulp and a soluble fraction containing modified hemicellulose monosaccharides and lignin was removed by filtration (Figure S2). Here, the initial goal was to optimise the butanosolv pretreatment for use with a herbaceous monocot agricultural waste product (Figure S3). ${ }^{[34]}$ The aim was to determine mild conditions capable of delivering rice husk butanosolv lignin (RHBL) in optimal yields with high retention of $\beta-\mathrm{O}-4$ content ( $\beta-\mathrm{O}-4$ per $100 \mathrm{C}$ unit calculation, Figure S5 and Table S2).

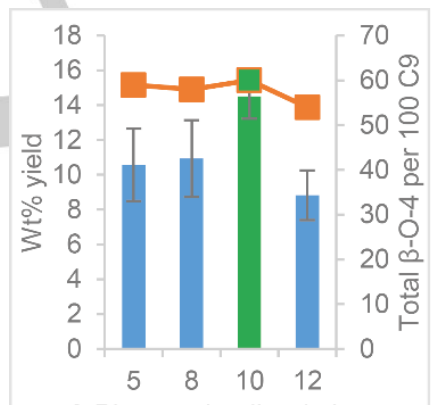

A Biomass loading ( $\mathrm{mL}$ solvent / $\mathrm{g}$ biomass)

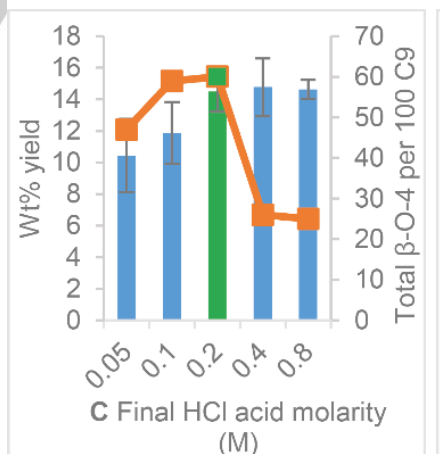

(M)

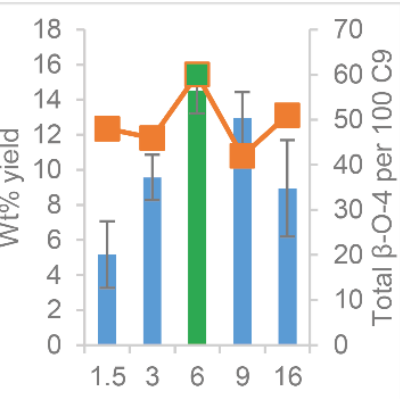

B Pretreatment time (h)

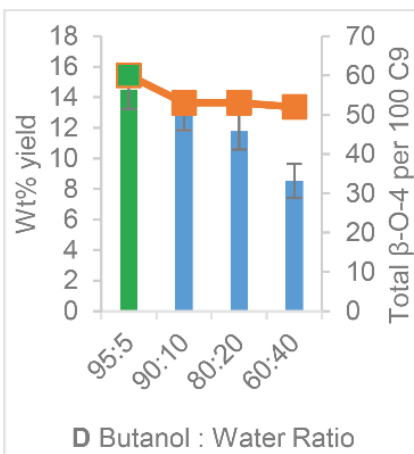

Figure 2. Graphs showing lignin weight $\%$ yields (bar charts) and total $\beta-O-4$ per 100 C9 data (line chart); A Effect of changing biomass loading (conditions identical except for quantity of solvent system used per gram of rice husks); B Pretreatment time (time pretreatment was maintained at reflux); C Final hydrochloric acid molarity in 95:5 butanol/water solvent system; D Ratio of butanol to water using a fixed final concentration of $0.2 \mathrm{M} \mathrm{HCl}$. The optimal conditions based on both lignin wt\% yields (green bars) and $\beta-O-4$ per $100 \mathrm{C} 9$ values are shown. All pretreatments were performed on a $4 \mathrm{~g}$ scale. Error bars are based on three repeats of each pretreatment. 
A range of biomass loadings were initially assessed varying from $5-12 \mathrm{~mL}$ of solvent per gram of rice husks. Whilst all the loadings led to the isolation of RHBL with a high total $\beta-O-4$ content, the lignin yield was optimal at $10 \mathrm{~mL} / \mathrm{g}$ (Figure 2A). Using this loading level, it was then shown that the reflux time also affected the lignin yield. The optimal outcome was obtained after 6 hours at reflux with yields dropping at extended times, presumably due to lignin condensation resulting in a significant proportion of the lignin being in the insoluble pulp (Figure 2B). Upon varying the acid molarity whilst retaining a butanol:water ratio of $95: 5$ (using $10 \mathrm{~mL} / \mathrm{g}$ loading and 6 hours at reflux), the highest yield of high quality RHBL was obtained when a final concentration of acid of $0.2 \mathrm{M}$ was used. A significant decrease in the $\beta-O-4$ content was observed when higher concentrations of acid were used (Figure $2 \mathrm{C}$ ) as expected. ${ }^{[13]}$ Finally, when the final acid concentration was fixed at $0.2 \mathrm{M}$, varying the butanol:water ratio showed that with increasing amounts of water relative to butanol, there was a decrease in yield (Figure 2D). Optimal conditions for the butanosolv pretreatment of rice husks were determined to be: biomass loading of $10 \mathrm{~mL} / \mathrm{g}, 6 \mathrm{~h}$ pretreatment time, $0.2 \mathrm{M}$ final concentration of hydrochloric acid in a $95: 5$ butanol:water ratio.

Gel permeation chromatography (GPC) analysis of RHBL was conducted during the optimisation study (Table S2). A general decrease in $M_{w}$ was observed with an increase in final acid molarity $\left(M_{w} 3939\right.$ for $0.05 M$ c.f. $M_{w} 2842$ for $\left.0.8 M\right)$ consistent with more acidic conditions leading to increased lignin cleavage and a reduction in chain length.

The scalability of the process was studied next. The quality of the RHBL was very similar over a pretreatment scale that ranged from $4 \mathrm{~g}$ to $400 \mathrm{~g}$ to $4000 \mathrm{~g}$. This was assessed using 2D HSQC NMR analysis and semi-quantitative integration of the spectra obtained for the different batches of RHBL (Figure 3, Table S3 and Figure S6). Yields of the different product streams were also reproducible across all the scales tested (Table S3).

The separation of the RHB lignin and the hemicellulosederived $(\mathrm{RHBH})$ streams using our published work-up protocol ${ }^{[15]}$ proved challenging on larger scale (Figures S1 and S7A-E). The use of the contrasting solubility of the lignin (insoluble) and the hemicellulose-derived streams (soluble) in aqueous solution was effective for small-scale pretreatments (Figure S7B) and small portions $(28 \mathrm{~g})$ of the crude hemicellulose-derived/lignin mixture retained from larger pretreatments (Figure S7C). However, attempts to process all the material from a $400 \mathrm{~g}$ pretreatment were hampered by very long filtration times after lignin precipitation (Figure S7D). The requirement to use large quantities of water for the lignin precipitation also meant that

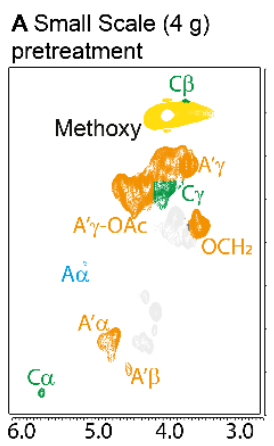

B Medium Scale (400 g) C Large Scale (4 kg)

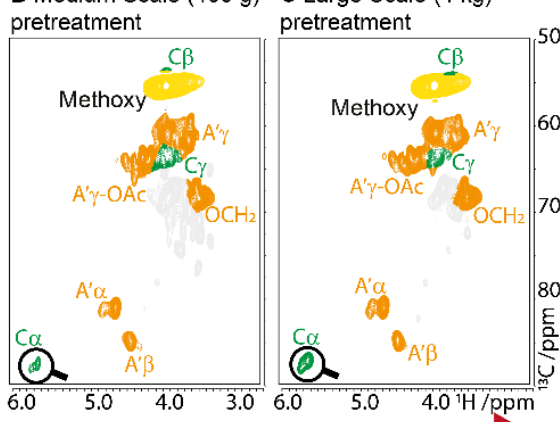

Increasing pretreatment scale

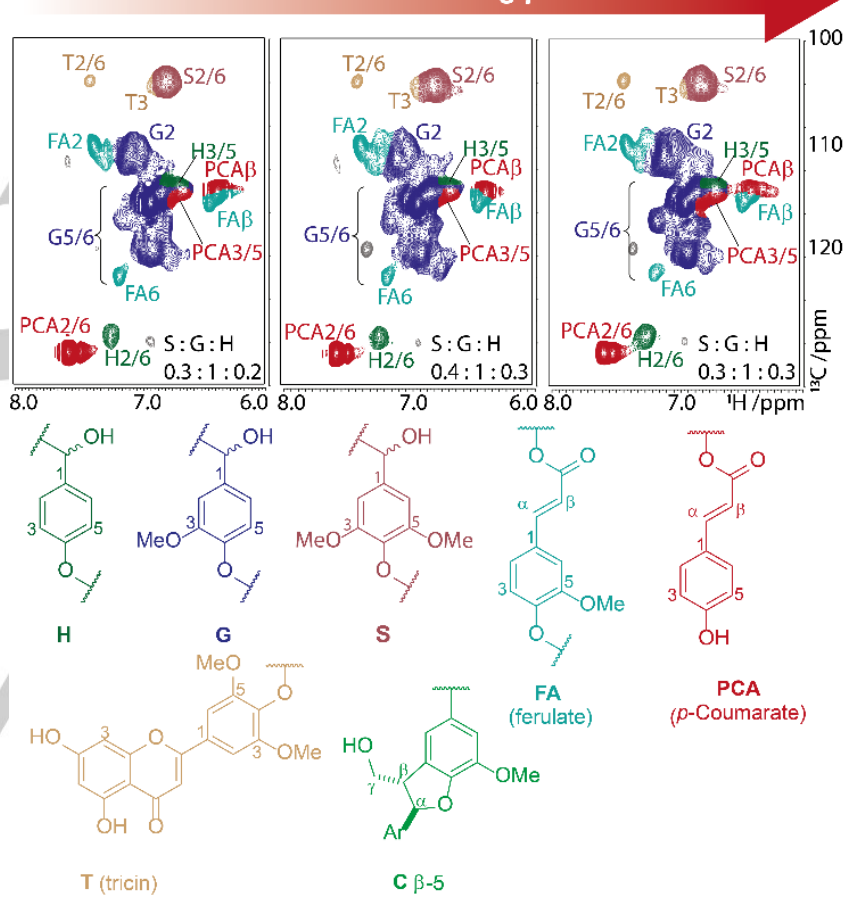

Figure 3. Sidechain and aromatic regions of 2D HSQC NMR spectra $(700 \mathrm{MHz}$ $\mathrm{d}_{6}$-Acetone) of extracted RHBL at different scales; A Small scale pretreatment (4 g rice husks); B Medium scale ( $400 \mathrm{~g}$ rice husks); C Large scale (4 kg rice husks). Colour palette used for different linkages, with the rest of the colour palette shown with Scheme 1. Magnification shown is x2 intensity.

obtaining the RHBH was tiresome (Figure S7D \& S7E). A modified work-up protocol was developed for large scale work (Figure S7A) in which the aqueous solution of the RHBH was first extracted with EtOAc giving separate water- and EtOAc-soluble $\mathrm{RHBH}$ streams. In one run, this led to $34 \%$ of the $\mathrm{RHBH}$ being in the EtOAc layer with the remainder being in the water. Furthermore, extended filtration times were avoided by centrifugation. 
In previous work with hardwood butanosolv lignins ${ }^{[15]}$ it was demonstrated that hemicellulose was depolymerised and chemically modified by butoxylation at the anomeric position of the monomeric sugars. In this work, the presence of specific sugar monomers was determined using 2D HSQC NMR analysis with spectra of authentic butoxylated xylose 4 , butoxylated glucose 5 and xylose 6 being used for comparison (Figure 4 and Figure S8A-G). The ethyl acetate-soluble stream was shown to be almost entirely comprised of butoxylated xylose 4 with some minor unknown contaminants (Figure 4). Whereas, the watersoluble stream was comprised of a mixture of native and butoxylated monosaccharides 4-7 (Figure S8) and some minor unidentified components. In an attempt to purify the mixture by flash column chromatography (using a $0-15 \% \mathrm{MeOH} / \mathrm{DCM}$

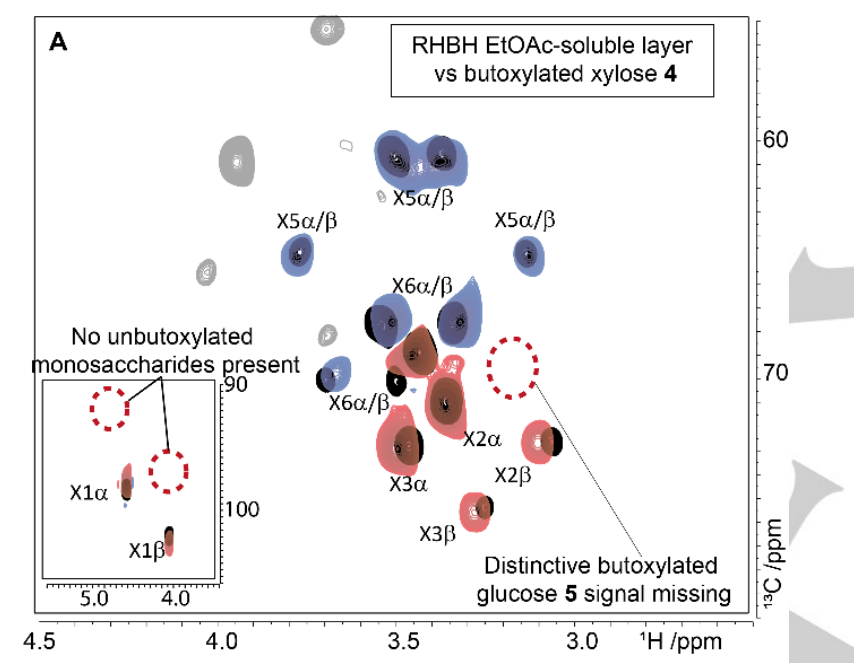

Figure 4. A Multiplicity edited $2 \mathrm{D}$ HSQC NMR analysis $\left(700 \mathrm{MHz}, \mathrm{D}_{2} \mathrm{O}\right)$ of EtOAc-soluble hemicellulose stream (blue and pick signals) overlaid with the spectra obtained on analysis of an authentic sample of $\alpha / \beta$-butoxylated xylose 4 (black). Multiplicity-edited cross-peaks corresponding to $\mathrm{CH}_{2}$ proton environments (blue) and multiplicity-edited cross-peaks corresponding to $\mathrm{CH}$ proton environments (red) shown. The anomeric region (inset box) highlights the absence of native monosaccharides (dashed regions). The absence of the distinctive $G 5 \alpha / \beta$ cross-peak observed in butoxylated glucose $\mathbf{5}$ is highlighted (dashed regions). B Reaction scheme showing preparation of authentic butoxylated monosaccharide standards $\mathbf{4}$ and $\mathbf{5}$. system), from $2 \mathrm{~g}$ of the crude lignin/hemicellulose mixture, $0.4 \mathrm{~g}$ of 4 and $0.2 \mathrm{~g}$ of $\mathbf{5}$ were obtained in excellent purity (Figure S9). Unfortunately, other native monosaccharides proved too polar to be eluted. Based on quantitative HSQC NMR analysis of the crude hemicellulose stream we estimate that the yields of butoxylated xylose $\mathbf{4}$ and glucose $\mathbf{5}$ are approximately 16 and 5 wt $\%$ respectively, and non-butoxylated xylose $6<2 \mathrm{wt} \%$, with respect to the starting biomass (Tables S4-S7 and Figures S13S15). Furthermore, the yield of butoxylated xylose 4 in the EtOAc extract was found to be approximately $4 \mathrm{wt} \%$, again with respect to the starting biomass (Table S7).

\section{TEMPO-mediated formation of $\beta-0-4 \quad y$-carboxylic acid- substituted butanosolv lignins}

In contrast to other $\mathrm{RH}$ product streams, ${ }^{[35-37]}$ the lignin has been understudied with the exception of its structural characterisation. ${ }^{[26,38,39]}$ It was therefore decided to assess if RHBL could be converted into a precursor for novel materials. Strategies for lignin modification often focus on the use of phenolic end groups and/or the unselective conversion of $\beta-O-4$ units by reaction at both the $\alpha$ - and $\gamma$-hydroxyls. In the case of butanosolv lignins, only the $\beta-O-4$-hydroxyl is substituted, ${ }^{[8]}$ providing an opportunity to carry out site selective oxidation of the $\mathrm{Y}$-hydroxyl to the corresponding $\mathrm{Y}$-carboxylic acid (to give lignin ${ }^{\gamma-}$ ${ }^{o x C A}$ ). It should be noted that a proportion of the $\beta-O-4$ units in RHBL are natively $Y$-acylated with sidechain esters such as $p$ coumarates (Figure S10) and that these $\gamma-\mathrm{Ac}-\beta-\mathrm{O}-4$ units would be inert to the planned oxidation reactions. ${ }^{[26,38,39]}$

Previous reports on $\beta-\mathrm{O}-4 \mathrm{\gamma}$-oxidation have focussed on the generation of aldehydes (lignin ${ }^{\gamma-O x A L D}$ ) in model systems and en route to lignin depolymerisation. ${ }^{[40,41]}$ To the best of our knowledge, only three reports describe the preparation of model $\beta-O-4 y-$ carboxylic acids and organosolv lignin ${ }^{\gamma-o x C A}$. All of these use TEMPO as the catalytic oxidant under electrochemical catalyst regeneration conditions. ${ }^{[42-44]}$ The initial report focussed on nonphenolic native $\beta-O-4$ models $^{[42]}$ and showed that the use of alkaline conditions was advantageous. Later, Stahl reported additional studies on the $\gamma$-oxidation of phenolic native $\beta-O-4$ models and concluded that, whilst cleavage reactions dominated quinone methide structures were formed under basic conditions with TEMPO. ${ }^{[43,44]}$ It seems clear that the presence of phenolic end-groups in lignin may well be problematic for the $\mathrm{Y}$-oxidation of $\beta-O-4$ unit. Indeed, Stahl indicated that using lignins with lower native phenolic content resulted in more efficient $\gamma$-oxidation. 

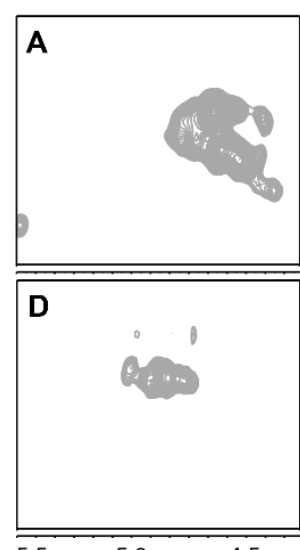

5.5

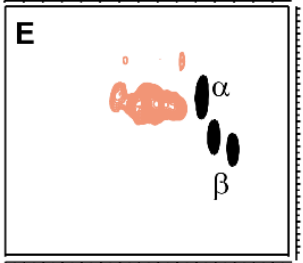

5.5

B

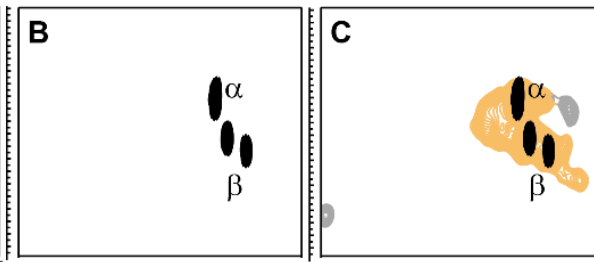

$\mathbf{F}$
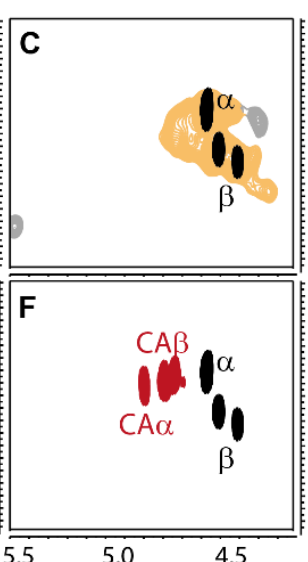

Figure 5. HSQC NMR analysis (700 MHz, d6-DMSO) of RHBLs; Signals corresponding to a selected region of the analysis of: A unmodified RHBL; B model 9; C overlay of model 8 and unmodified RHBL; D RHBL ${ }^{\text {r-oxCA }}$ (prepared using general procedure $\mathrm{C}$ ); $\mathbf{E}$ overlay of $\mathrm{RHBL}^{\mathrm{r}-\mathrm{oxCA}}$ and model $\mathbf{8} ; \mathbf{F}$ overlay of model 8 (black) and model $\mathbf{9}$ (red); G overlay of RHBL ${ }^{r-o x C A}$ and model $\mathbf{9}$; $\mathbf{H}$ $\delta^{1} \mathrm{H} / \delta^{13} \mathrm{C} 9-10 \mathrm{ppm} / 190-210$ ppm region in RHBL $\mathrm{L}^{\mathrm{r}-\mathrm{oxCA}}$ highlighting the absence of an aldehyde signals (dashed red region). For a full labelled RHBL ${ }^{\mathrm{Y}-\mathrm{x} C A}$ spectrum, refer to Figure S11A.

Despite the fact that lignin from grasses is known to have a high native phenolic content due to coumarates and ferulates, it was decided to attempt to convert our RHBL to RHBL ${ }^{\text {-oxCA }}$ using a TEMPO/NCS system that had previously been reported to oxidise relatively simple primary alcohols. ${ }^{[45]}$

Encouragingly, butoxylated $\beta-\mathrm{O}-4$ model 8 was oxidised allowing isolation of $\gamma$-acid model 9 (Schemes 1 and S2). Subsequent formation of the $\beta-\mathrm{O}-4 \mathrm{\gamma}$-carboxylic acid in RHBL was observed when the reaction was run with catalytic quantities of TEMPO at $\mathrm{pH} \mathrm{9-10} \mathrm{for} \mathrm{extended} \mathrm{reaction} \mathrm{times} \mathrm{(Scheme} \mathrm{S1,}$ general procedure $\mathrm{C}$ ) with a significant excess of NCS being used as a co-oxidant. Noticeable changes in the chemical shift of the cross-peaks corresponding to the butoxylated $\beta-0-4$ unit were seen upon $\gamma$-oxidation (from $\delta^{1} \mathrm{H} / \delta^{13} \mathrm{C} 4.5 \mathrm{ppm} / 82 \mathrm{ppm}$ for the $\alpha$ proton and $4.4 \mathrm{ppm} / 83 \mathrm{ppm}$ of the unreacted unit to $4.7 \mathrm{ppm} / 82$ $\mathrm{ppm}$ for the $\alpha$-proton and $4.6 \mathrm{ppm} / 81 \mathrm{ppm}$ for the $\beta$-proton of the $\gamma$-oxidised unit, Figure 5). Evidence for the successful formation of RHBL ${ }^{\mathrm{Y}-\mathrm{oxCA}}$ also came from good overlay with signals from the relevant model 9 (Figure $5 \mathrm{G}$ ) and not the unoxidised model 8 (Figure $5 \mathrm{E}$ and $5 \mathrm{G}$ ). The formation of $\mathrm{RHBL}^{\mathrm{y}-\mathrm{oxCA}}$ occurred selectively under these conditions with no detectable formation of RHBL ${ }^{\text {Y-oxALD }}$ (absence of signals corresponding to aldehyde environments in $\mathrm{RHBL}{ }^{\mathrm{r}-\mathrm{oxCA}}$, Figure $5 \mathrm{H}$ inset).

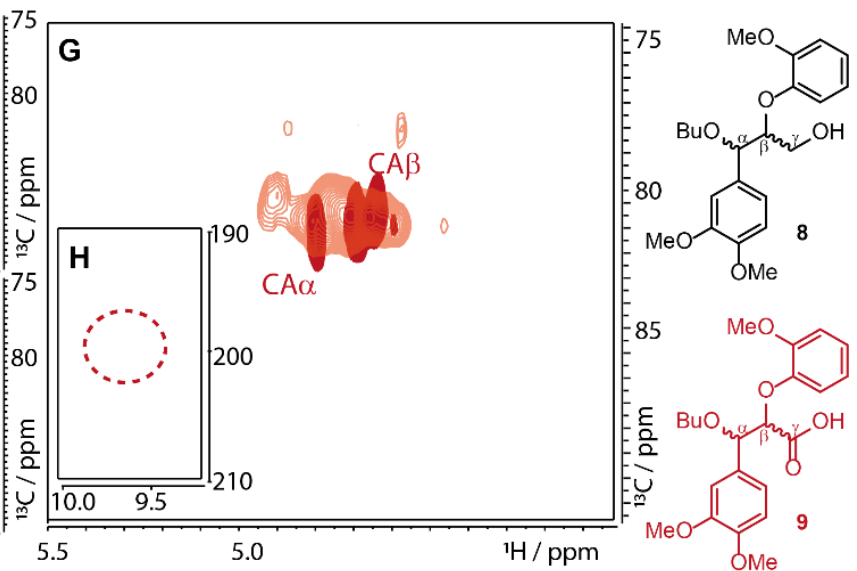

Quantitative ${ }^{31} \mathrm{P}$ NMR analysis was also supportive of a significant increase in carboxylic acid environments in the RHBL ${ }^{r-}$ oxCA compared to unoxidised RHBL (Figure S12). However, there was a significant loss of signals corresponding to phenols, potentially consistent with the previously reported quinone methide formation. ${ }^{[43,44]}$ The analogous $y$-oxidation of other butanosolv lignins was conducted and demonstrated that this approach was generally applicable. Walnut shell butanosolv lignin (WSBL, from a hardwood source, Figure 1 and Figure S4C), buckwheat butanosolv lignin (BWBL, hardwood, Figure S4K) and Douglas fir butanosolv lignin (DFBL, softwood, Figure S4I) were all successfully converted to the corresponding $\mathrm{Y}$-carboxylic acidcontaining materials (Figure S11).

With RHBL ${ }^{\mathrm{r}-\mathrm{oxCA}}$ in hand, it was decided to couple the newly introduced carboxylic acids with aniline $\mathbf{1 0}$, as it was felt this represented a flexible approach to grafting onto the lignin backbone that complemented other recent reports by us and others. ${ }^{[5,46-52]}$ Initially, model 11 was synthesised from $\mathbf{9}$ and $\mathbf{1 0}$ to aid characterisation of the final lignin (Scheme 1). RHBL ${ }^{\gamma-o x C A}$ was then coupled with 10 using EDC, $\mathrm{Et}_{3} \mathrm{~N}$ and $\mathrm{HOBt}$ at rt for 24 hours (Lignin general procedure D). ${ }^{[53]}$ The resulting lignin was characterised using HSQC NMR analysis (Figure 6) and compared with model 11 (Figure 6A). Aniline 10 was selected for these studies as on formation of the lignin-aniline amide bond a distinctive change in the chemical shift of the aniline aromatic protons was expected. Comparison of the signals observed for the $y$-coupled lignin with those of aniline 10 and acetylated aniline 12 (and model 11) supported the formation of the desired amide bond in the lignin (Figures $6 \mathrm{~A}$ and $6 \mathrm{~B}$ ). As expected, the crosspeaks corresponding to the aromatic protons in aniline $\mathbf{1 0}$ $\left(\delta^{1} \mathrm{H} / \delta^{13} \mathrm{C} 7.0 \mathrm{ppm} / 129 \mathrm{ppm}, 6.5 \mathrm{ppm} / 116 \mathrm{ppm}\right.$ and $6.6 \mathrm{ppm} / 114$ $\mathrm{ppm}$ ) were very distinct from the cross-peaks in acetylated aniline 
<smiles>COc1cccc(C(O)(O)C(O)(O)c2ccccc2OC)c1</smiles>

TEMPO (0.1 eq.), NCS (10 eq.), TBAB,

$(1: 1) \mathrm{DCM} /$ aq. $\mathrm{NaHCO}_{3}(\mathrm{pH} 9.5)$ rt, $24 \mathrm{~h}$<smiles>COc1cccc(OC(=O)C(OC(=O)O)c2ccccc2OC)c1</smiles><smiles>COc1ccccc1OC(c1ccccc1OC)C(Oc1ccccc1)C(=O)Nc1ccccc1</smiles>

Scheme 1. Preparation of butoxylated $y$-acid $\beta$-O-4 model 9 and $\mathrm{EDC} / \mathrm{HOBt} / \mathrm{Et}_{3} \mathrm{~N}$ amide coupling with aniline 10 to give $\gamma$-coupled model 11.

$12\left(\delta^{1} \mathrm{H} /{ }^{13} \mathrm{C} 7.6 \mathrm{ppm} / 119 \mathrm{ppm}, 7.3 \mathrm{ppm} / 129 \mathrm{ppm}\right.$ and $7.0 \mathrm{ppm} / 123$ ppm), model 11 and in the coupled RHBL ${ }^{\mathrm{y} \text {-amide }}$. There was a clear appearance of new aniline-related aromatic cross peaks that were not present in the starting RHBL $^{\mathrm{r}-\mathrm{oxCA}}$ (Figure 6C).

\section{Conclusions}

The butanosolv pretreatment process was applied to rice husks with variations in a number of the reaction parameters being carried out. Optimal conditions were determined to be a $10 \mathrm{~mL} / \mathrm{g}$ solvent to biomass loading, in a 95:5 ratio of butanol to water at a final acid molarity of $0.2 \mathrm{M}$ for a pretreatment time of $6 \mathrm{~h}$ at reflux. This process was shown to be efficient on a variety of scales, with repeatably good conversion of biomass into high quality product streams on scales of up to $4 \mathrm{~kg}$ of biomass. Pure butoxylated xylose (anomeric mixture) was isolated directly from the pretreatment in high yield based on the amount of xylose present in the starting biomass. In addition, selective $\mathrm{Y}$-oxidation of the butoxylated $\beta-O-4$ units in the butanosolv rice husk lignin gave $\gamma$ carboxylic acids despite the presence of phenolic end groups in the lignin. Evidence for the success of this selective lignin oxidation protocol came from detailed $\mathrm{HSQC}$ and ${ }^{31} \mathrm{P}$ NMR analysis including comparison with relevant model compounds. However, preparation of $\mathrm{RHBL}^{\mathrm{r}-\mathrm{oxCA}}$ required a significant amount of the co-oxidant NCS and the system is relatively complex. Future work will strive to reduce the complexity of the oxidation system.

Finally, the oxidised lignin was used in an exemplar amide coupling reaction leading to an amide-grafted lignin. The potential for the controlled preparation of a range of complex lignins via this overall approach is considerable.

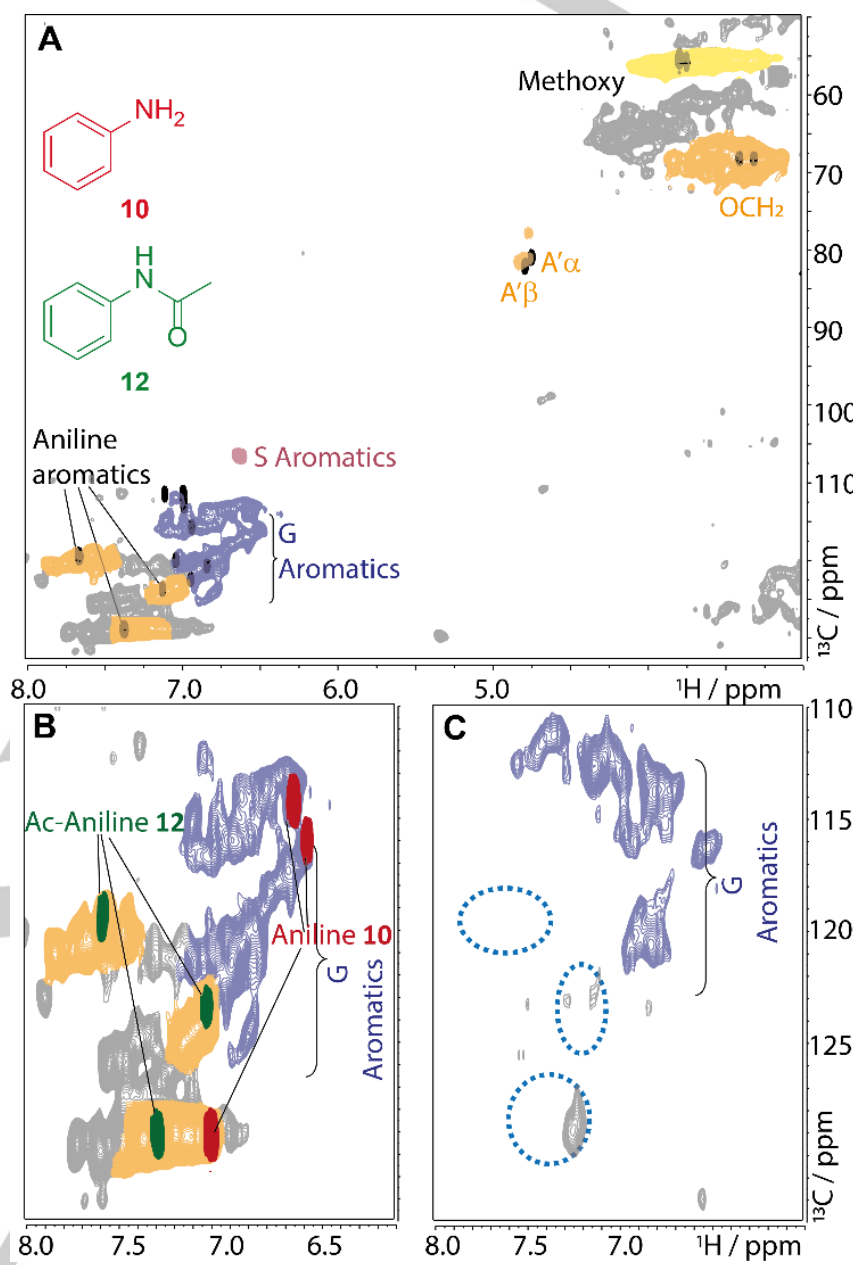

Figure 6: HSQC NMR analysis (700 MHz, $\mathrm{d}_{6}$-DMSO) of $\mathrm{RHBL}^{\mathrm{r} \text {-amide }}$. A spectrum of $y$-coupled RHBL overlaid with spectrum of model 11 (black). Environments corresponding to $\gamma$-coupled $\beta-O-4$ protons are coloured in orange. $\mathbf{B}$ aromatic region from analysis of $\gamma$-coupled RHBL overlaid with spectra of aniline 10 (red) and acetylated aniline $\mathbf{1 2}$ (green). $\mathbf{C}$ aromatic region from analysis of starting $\mathrm{RHBL}^{\mathrm{r}-\mathrm{oxCA}}$ highlighting absence of signals corresponding to aniline aromatic environments are clearly absent (highlighted in blue dashed circles).

\section{Experimental Section}

All chemicals used in this report were obtained from commercial sources Rice husks biomass was obtained from The Home Brew Shop, walnut shell biomass was a kind donation from Sharpham Park farm, and all other biomasses were received from Hot Smoked. Full characterisation data consisting of ${ }^{1} \mathrm{H} \mathrm{NMR},{ }^{13} \mathrm{C} N M R, 2 \mathrm{D} H \mathrm{HQC} N M R, I R$ and HRMS are provided for all novel compounds (refer to ESI).

\section{Lignin General Procedure A: Butanosolv Pretreatment}

Following a literature procedure, ${ }^{[15]}$ to unextracted biomass was added $n$ butanol: $0.2 \mathrm{M} \mathrm{HCl}(95: 5,10 \mathrm{~mL} / \mathrm{g})$. The mixture was heated at a gentle reflux (ca. $100^{\circ} \mathrm{C}$ ) for $6 \mathrm{~h}$ and vacuum filtered when cooled. The residual 
pulp was washed with a solution of acetone: $\mathrm{H}_{2} \mathrm{O}$ (9:1), and the resultant filtrate was concentrated in vacuo to yield a gum-like residue (azeotrope with additional portions of water to remove all butanol). The residual pulp (cellulose-enriched pulp) was dried in a vacuum oven overnight at $50^{\circ} \mathrm{C}$. The gum-like residue was dissolved in the minimum amount of acetone: $\mathrm{H}_{2} \mathrm{O}(9: 1,5 \mathrm{~mL} / \mathrm{g})$ and added dropwise to rapidly stirring $\mathrm{H}_{2} \mathrm{O}(50$ $\mathrm{mL} / \mathrm{g}, 10 \mathrm{v} / \mathrm{v}$ minimum). A small portion of sat. sodium sulfate solution added $(1 \mathrm{~mL} / 100 \mathrm{~mL}$ water). The resulting crude precipitate was collected by vacuum filtration, or centrifugation at $8000 \mathrm{rpm}$, and dried in a vacuum oven at $50^{\circ} \mathrm{C}$ for $16 \mathrm{~h}$.

\section{Lignin General Procedure B: Phosphitylation and ${ }^{31} \mathrm{P}$ NMR analysis of Lignin}

All lignins were dried for $16 \mathrm{~h}$ at $50{ }^{\circ} \mathrm{C}$ in a vacuum oven before measurements. $30 \mathrm{mg}$ of lignin was weighed into a vial and dissolved in $0.5 \mathrm{~mL}$ of $\mathrm{CDCl}_{3} /$ pyridine (1:1.6, anhydrous, dried over $4 \AA \mathrm{MS}$ ). Following a literature procedure, ${ }^{[54]}$ to this solution was added $10 \mu \mathrm{L}$ cyclohexanol (dried over $4 \AA \mathrm{MS}$ ) and $50 \mu \mathrm{L}$ 2-chloro-4,4,5,5-tetramethyl-1,3,2dioxaphospholane. The solution was transferred to a clean, dry NMR tube, purged with $\mathrm{N}_{2}$ and sealed, then analysed using quantitative ${ }^{31} \mathrm{P}$ NMR analysis within $6 \mathrm{~h}$ of sample prep.

\section{Lignin General Procedure C: TEMPO/NCS y-Oxidation to Butanosolv Lignin $^{\mathrm{r}-\mathrm{oxCA}}$}

To a solution of butanosolv lignin $(0.4 \mathrm{~g})$ and TBAB $(0.03 \mathrm{~g})$ in a DCM/aq. buffered system (1:1, $2 \mathrm{~mL}$, aq. sat. $\mathrm{NaHCO}_{3}, \mathrm{pH}$ 9-10) was added NCS $(1 \mathrm{~g})$ and TEMPO $(0.12 \mathrm{~g})$. The biphasic reaction mixture was stirred rapidly (ensuring layer mixing) at it for $24 \mathrm{~h}$. The reaction mixture was concentrated in vacuo until only the aqueous layer remained. The aqueous layer was decanted into rapidly stirring water $(200 \mathrm{~mL} / \mathrm{g})$, and the lignin precipitate remaining in the flask was dissolved in acetone $(10 \mathrm{~mL} / \mathrm{g})$ and also added dropwise into the rapidly stirring water. To the stirring mixture was added conc. $\mathrm{HCl}$ (approximately $20 \mathrm{drops} / \mathrm{g}$ ), until the lignin was observable as a precipitate. The lignin precipitate was filtered off and dried in a vacuum oven for $16 \mathrm{~h}$ at $50^{\circ} \mathrm{C}$ to give a brown powder.

Lignin General Procedure D: Y-Coupling of Butanosolv Lignin ${ }^{\text {v-oxCA }}$

To a solution of butanosolv lignin ${ }^{--o x C . A}(0.4 \mathrm{~g})$, HOBt $(0.14 \mathrm{~g})$ and EDC $(0.14 \mathrm{~mL})$ in DCM $(15 \mathrm{~mL})$ was added the amine coupling partner $(2 \mathrm{mmol})$ and stirred at rt for $1 \mathrm{~h}$. To the reaction mixture was added $\mathrm{Et}_{3} \mathrm{~N}(0.32 \mathrm{~mL})$ and stirred at it for $16 \mathrm{~h}$. The reaction mixture was concentrated in vacuo, then dissolved in the minimum amount of acetone (approximately $20 \mathrm{~mL} / \mathrm{g}$ of starting lignin) and added dropwise into rapidly stirring water $(200 \mathrm{~mL} / \mathrm{g}$. The precipitated lignin was filtered off and washed extensively with additional water, then dried in a vacuum oven for $16 \mathrm{~h}$ at $50^{\circ} \mathrm{C}$ to give a brown powder.

\section{Acknowledgements}

We would like to thank the CRITICAT Centre for Doctoral Training for financial support [Ph.D. studentship to IP; Grant code: EP/L016419/1] and BBSRC Global Challenges Research Fund Impact Acceleration Account at St Andrews BB/GCRFIAA/20. CSL thanks the Leverhulme Trust for funding an Early Career Fellowship. We acknowledge the EPSRC UK National Mass Spectrometry Facility at Swansea University for mass spectrometry analysis, Sharpham Park farms for their kind donation of walnut shells and Dr Daniel Miles-Barrett for helpful discussions.

Keywords: Sustainable Chemistry • Biomass • Oxidation • Green Chemistry • Lignin

[1] P. J. Deuss, K. Barta, Coord. Chem. Rev. 2015, 510-532.

[2] A. Berlin, M. Balakshin, Industrial Lignins, Elsevier, 2014.

[3] H. Vappula, Pulp Market Review - Energy and Pulp Business Group. 2011.

[4] J. Zakzeski, P. C. a Bruijnincx, A. L. Jongerius, B. M. Weckhuysen Chem. Rev. 2010, 110, 3552-99.

[5] I. Panovic, J. R. D. Montgomery, C. S. Lancefield, D. Puri, T. Lebl, N J. Westwood, ACS Sustain. Chem. Eng. 2017, 5, 10640-10648.

[6] N. J. Westwood, I. Panovic, C. S. Lancefield, Springer Singapore, 2016, pp. $183-216$

[7] C. S. Lancefield, O. S. Ojo, F. Tran, N. J. Westwood, Angew. Chemie Int. Ed. 2015, 54, 258-262.

[8] P. J. Deuss, M. Scott, F. Tran, N. J. Westwood, J. G. de Vries, K Barta, J. Am. Chem. Soc. 2015, 137, 150522131446003.

[9] A. Rahimi, A. Ulbrich, J. J. Coon, S. S. Stahl, Nature 2014, 515, 249252.

[10] D. M. Miles-Barrett, A. R. Neal, C. Hand, J. R. D. Montgomery, I. Panovic, O. S. Ojo, C. S. Lancefield, D. B. Cordes, A. M. Z. Slawin, T. Lebl, et al., Org. Biomol. Chem. 2016, 14, 10023-10030.

[11] C. S. Lancefield, G. M. M. Rashid, F. Bouxin, A. Wasak, W.-C. Tu, J. P. Hallett, S. Zein, J. Rodriguez, S. D. Jackson, N. J. Westwood, et al., ACS Sustain. Chem. Eng. 2016, 4, 6921-6930.

[12] S. Constant, H. L. J. Wienk, A. E. Frissen, P. de Peinder, R. Boelens, D. S. van Es, R. J. H. Grisel, B. M. Weckhuysen, W. J. J. Huijgen, R. J. A. Gosselink, et al., Green Chem. 2016, 18, 2651-2665.

[13] S. Bauer, H. Sorek, V. D. Mitchell, A. B. Ibáñez, D. E. Wemmer, J. Agric. Food Chem. 2012, 60, 8203-8212.

[14] G. Hu, C. Cateto, Y. Pu, R. Samuel, A. J. Ragauskas, Energy and Fuels 2012, 26, 740-745.

[15] C. S. Lancefield, I. Panovic, P. J. Deuss, K. Barta, N. J. Westwood, Green Chem. 2016, 19, 1203-1210.

[16] a. a. Pereira, G. F. Martins, P. a. Antunes, R. Conrrado, D. Pasquini, a. E. Job, a. A. S. Curvelo, M. Ferreira, a. Riul, C. J. L. Constantino, Langmuir 2007, 23, 6652-6659.

[17] H. Teramura, K. Sasaki, T. Oshima, F. Matsuda, M. Okamoto, T. Shirai, H. Kawaguchi, C. Ogino, K. Hirano, T. Sazuka, et al., Biotechnol. Biofuels 2016, 9, 1-11.

[18] L. F. Del Rio, R. P. Chandra, J. N. Saddler, Appl. Biochem. Biotechnol. 2009, 161, 1-21.

[19] K. Wang, H. Yang, S. Guo, X. Yao, R.-C. Sun, J. Appl. Polym. Sci. 2014, 131, n/a--n/a.

[20] K. Wang, H. Yang, S. Guo, Y. Tang, J. Jiang, F. Xu, R.-C. Sun, Process Biochem. 2012, 47, 1503-1509.

[21] T. Renders, S. Van den Bosch, S.-F. Koelewijn, W. Schutyser, B. F. Sels, Energy Environ. Sci. 2017, 10, 1551-1557.

[22] L. Shuai, M. T. Amiri, Y. M. Questell-Santiago, F. Héroguel, L. Yanding, H. Kim, R. Meilan, C. Chapple, J. Ralph, J. S. Luterbacher, 
et al., Science (80-. ). 2016, 354, 329-334.

[23]

W. Lan, M. Talebi Amiri, C. M. Hunston, J. Luterbacher, Angew. Chemie Int. Ed. 2017, 5, 1356-1360.

[24] F. Stat, "FAO Stat," 2013.

[25]

S. Chandrasekhar, K. G. Satyanarayana, P. N. Pramada, P. Raghavan, T. N. Gupta, J. Mater. Sci. 2003, 38, 3159-3168.

A. Salanti, L. Zoia, M. Orlandi, F. Zanini, G. Elegir, J. Agric. Food Chem. 2010, 58, 10049-10055

[27]

[28]

[29]

[30]

[32]

R. Pode, Renew. Sustain. Energy Rev. 2016, 53, 1468-1485.

R. Saad, J. Hawari, J. Porous Mater. 2012, 20, 227-233.

F. Adam, T. S. Chew, J. Andas, J. Sol-Gel Sci. Technol. 2011, 59, 580-583.

Z. Wang, J. Yu, X. Zhang, N. Li, B. Liu, Y. Li, Y. Wang, W. Wang, Y. Li, L. Zhang, et al., ACS Appl. Mater. Interfaces 2016, 8, 1434-1439. J. Umeda, K. Kondoh, Ind. Crops Prod. 2010, 32, 539-544.

H. Chen, W. Wang, J. C. Martin, A. J. Oliphant, P. A. Doerr, J. F. Xu, K. M. DeBorn, C. Chen, L. Sun, ACS Sustain. Chem. Eng. 2013, 1, 254-259. Mater. Interfaces 2012, 4, 977-981.

H. Zhang, Y. Luo, L. Wu, Y. Huang, P. Christie, Environ. Sci. Pollut. Res. 2015, 22, 5908-5918.

B. C. Saha, L. B. Iten, M. a Cotta, Y. V Wu, Biotechnol Prog 2005, 21, 816-822.

[36]

N. Johar, I. Ahmad, A. Dufresne, Ind. Crops Prod. 2012, 37, 93-99.

D. Nabarlatz, A. Ebringerová, D. Montané, Carbohydr. Polym. 2007,

69, 20-28.

[38]

S. K. Singh, P. L. Dhepe, Bioresour. Technol. 2016, 221, 310-317.

[39]
Prod. 2013, 50, 887-895.

[40] S. Dabral, Ø. G. Hern, P. C. J. Kamer, C. Bolm, ChemSusChem 2017 7, 2707-2713.

[41] C. S. Lancefield, L. W. Teunissen, B. M. Weckhuysen, P. C. Bruijnincx, Green Chem. 2018, 19, DOI 10.1039/c7gc00195a

[42] Y. Sannami, H. Kamitakahara, T. Takano, Holzforschung 2017, 71, 109-117.

[43] S. S. Stahl, US20170342574A1, 2017

[44] S. S. Stahl, US20170342219A1, 2017

[45] J. Einhorn, C. Einhorn, F. Ratajczak, J.-L. Pierre, J. Org. Chem. 1996, $61,7452-7454$.

[46] C. Jin, X. Zhang, J. Xin, G. Liu, G. Wu, Z. Kong, J. Zhang, ACS Sustain. Chem. Eng. 2017, 5, 4086-4093.

[47] H. Liu, H. Chung, Macromolecules 2016, 49, 7246-7256.

[48] K. Xiong, C. Jin, G. Wu, G. Liu, Z. Kong, Chem. Ind. For. Prod. 2016 36, 115-120.

[49] Y. Han, L. Yuan, G. Li, L. Huang, T. Qin, F. Chu, Polymer (Guildf) 2016, 83, 92-100.

[50] C. Wang, R. A. Venditti, ACS Sustain. Chem. Eng. 2015, 3, 18391845.

[51] Pietro Buono, L. Averous, A. Duval, Y. Habibi, ChemSusChem 2018 11, 2472-2491.

K. Isozaki, T. Shimoaka, S. Oshiro, A. Yamaguchi, F. Pincella, R. Ueno, T. Hasegawa, T. Watanabe, H. Takaya, M. Nakamura, ACS Omega 2018, 3, 7483-7493.

M. Tsakos, E. S. Scha, L. L. Clement, N. L. Villadsen, T. B. Poulsen, Nat. Prod. Rep. 2015, 32, 605-632.

[54] A. Granata, D. S. Argyropoulos, J. Agric. Food Chem. 1995, 43 1538-1544. 


\section{Entry for the Table of Contents}

\section{FULL PAPER}

Rice husks were extracted efficiently using butanol as a pretreatment solvent on a variety of scales ( $4 \mathrm{~g}$ to 4 $\mathrm{kg}$ ) giving a cellulose-derived pulp, butanol-modified hemicellulose monosaccharides and a butanolmodified lignin. This butanosolv lignin was selectively oxidised at the $y$ position to give a carboxylic acid and tested in a coupling reaction with aniline, demonstrating a new approach for the preparation of lignin grafted materials.
I. Panovic, D. Phillips, M. J. Gronnow, N. J. Westwood*

Page No. 1 - Page No. 8

Selective Primary Oxidation of Lignin Streams from Butanol-Pretreated Agricultural Waste Biomass 